\title{
Criticality of Confined Fluids Based on the Tensile Strength of Liquids
}

\author{
Sugata P. Tan*, Elizabeth Barsotti, Mohammad Piri
}

Center of Innovation for Flow through Porous Media, Department of Petroleum Engineering, University of Wyoming, 1000 E. University Ave., Laramie, WY 82071, U.S.A.

*Email: sptan@uwyo.edu

\section{PC-SAFT parameters}

Table S1. PC-SAFT parameters, critical temperature $\left(T_{\mathrm{C}}\right)$, and LJ molecular diameter $(\sigma)$

\begin{tabular}{|l|c|c|c|c|c|c|}
\hline Pure Compound & $m$ & $\sigma[\AA]$ & $\varepsilon / k[\mathrm{~K}]$ & Reference & $T_{\mathrm{C}}[\mathrm{K}]^{*}$ & $\sigma[\mathrm{nm}]^{* *}$ \\
\hline Nitrogen & 1.2414 & 3.2992 & 89.2230 & TKM, 2013 & 126.19 & 0.3850 \\
\hline Argon & 1.0000 & 3.3768 & 117.8538 & TKM, 2013 & 150.69 & 0.3408 \\
\hline Oxygen & 1.1621 & 3.1661 & 112.7862 & TP, 2015 & 154.58 & 0.3504 \\
\hline Carbon dioxide & 2.5834 & 2.5564 & 151.7666 & TP, 2015 & 304.13 & 0.4185 \\
\hline Ethane & 1.6069 & 3.5206 & 191.4200 & GS, 2001 & 305.33 & 0.4384 \\
\hline Propane & 2.0020 & 3.6184 & 208.1100 & GS, 2001 & 369.82 & 0.5240 \\
\hline$n$-butane & 2.3316 & 3.7086 & 222.8800 & GS, 2001 & 425.12 & 0.5869 \\
\hline$n$-pentane & 2.6747 & 3.7656 & 232.1710 & TP, 2015 & 469.70 & 0.6099 \\
\hline$n$-hexane & 3.0127 & 3.8075 & 238.7873 & TP, 2015 & 507.82 & 0.5916 \\
\hline$n$-heptane & 3.4831 & 3.8049 & 238.4000 & GS, 2001 & 540.13 & 0.8880 \\
\hline
\end{tabular}

* NIST Webbook Chemical Web Book (Lemmon et al)

** Hirschfelder et al. (1964); taken as the average if more than one value is listed in the reference.

\section{Performance of PC-SAFT}

Within the temperature ranges relevant to this work, the EOS performance in describing the saturated liquid phase of pure compounds discussed in this study is shown in the Figure S1.
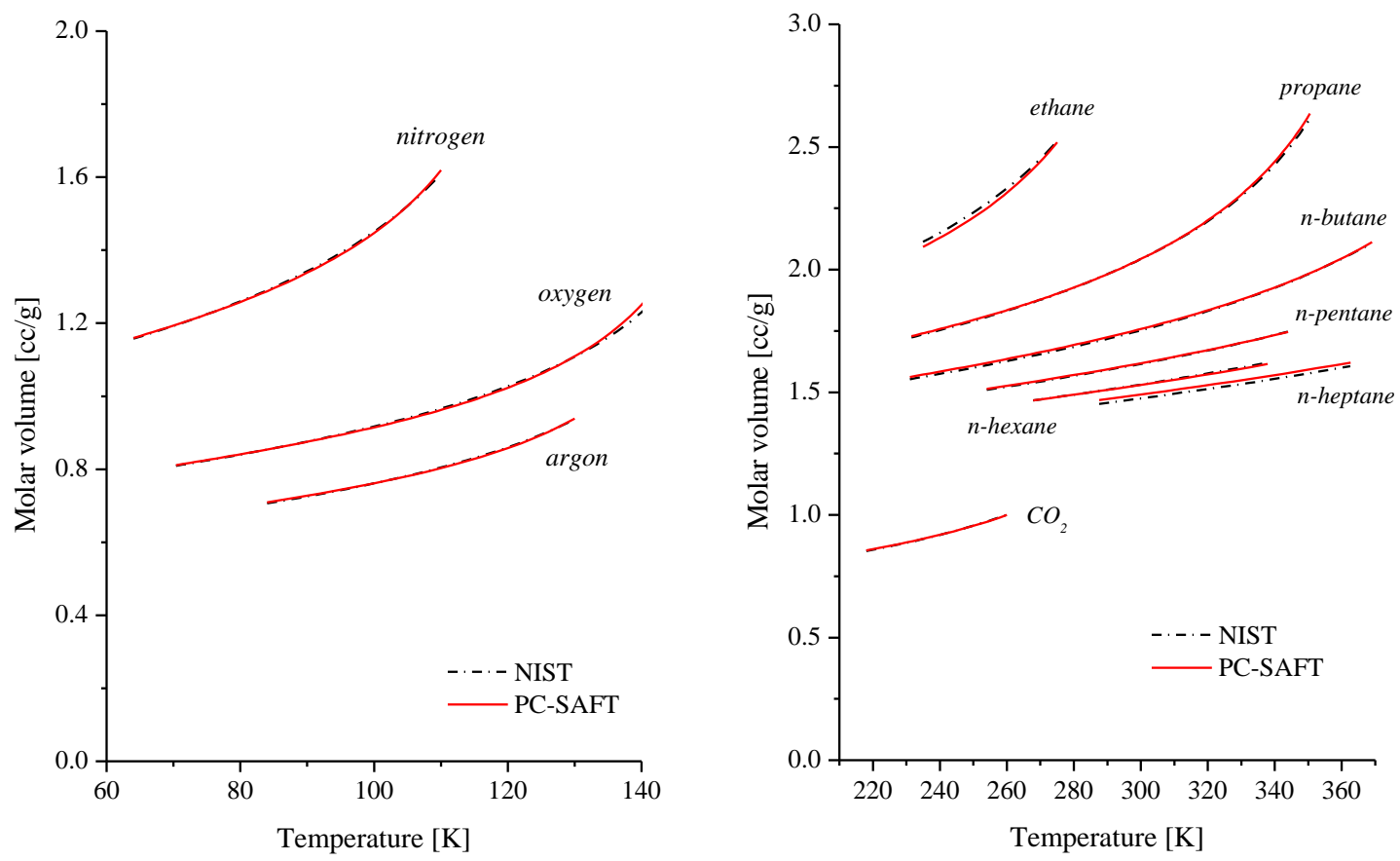

Figure S1. Performance of PC-SAFT for the saturated liquid phase using the parameters in Table S1. 
NIST data are taken from NIST Chemical Web Book (Lemmon et al). The parameters of PCSAFT are taken from Tan \& Piri (2015) except for ethane, propane, $n$-butane, and $n$-heptane, which are taken from Gross \& Sadowski (2001). It can be seen from the figures that calculations using PC-SAFT are sufficiently accurate for the purpose in this study. The average deviations for molar volume of saturated liquid calculated using PC-SAFT relative to NIST data are: $0.19 \%\left(\mathrm{~N}_{2}\right), 0.30 \%\left(\mathrm{O}_{2}\right), 0.20 \%(\mathrm{Ar})$, $0.77 \%\left(\mathrm{C}_{2} \mathrm{H}_{6}\right), 0.25 \%\left(\mathrm{C}_{3} \mathrm{H}_{8}\right), 0.32 \%\left(n \mathrm{C}_{4} \mathrm{H}_{10}\right), 0.16 \%\left(n \mathrm{C}_{5} \mathrm{H}_{12}\right), 0.15 \%\left(n \mathrm{C}_{6} \mathrm{H}_{14}\right)$, and $1.0 \%\left(n \mathrm{C}_{7} \mathrm{H}_{16}\right)$.

\section{Correction parameters}

The correction parameters used to reproduce the capillary-condensation pressures in systems of $n$-butane in 4.19-nm MCM-41 and argon at $87 \mathrm{~K}$ in MCM-41, as shown in Figure 15, are as follows:

n-butane in 4.19-nm MCM-41:

$$
\lambda(T)=11.185377-0.083266 T+1.611258 \times 10^{-4} T^{2}
$$

argon at $87 \mathrm{~K}$ in MCM-41:

$$
\lambda\left(R_{\mathrm{p}}\right)=-0.041936+1.139321 / R_{\mathrm{p}}
$$

where $\lambda$ is the correction parameter, $T$ is the absolute temperature, and $R_{\mathrm{p}}$ is the pore physical radius, which is related to the effective radius in eq 1 in the main paper according to: $r_{\mathrm{p}}=R_{\mathrm{p}}(1-\lambda)$. The correlation equations S1 and S2 were both derived from the respective experimental data (see the data sources listed in Table 1 in the main paper).

\section{References}

Lemmon, E.W.; McLinden, M.O.; Friend, D.G. Thermophysical Properties of Fluid Systems. In NIST Chemistry WebBook, NIST Standard Reference Database Number 69 [Online]; Linstrom, P.J.; Mallard, W.G., Eds.; National Institute of Standards and Technology: Gaithersburg, MD, USA; https://doi.org/10.18434/T4D303 (accessed March 21, 2019).

Gross, J.; Sadowski, G. Perturbed-Chain SAFT: An Equation of State Based on a Perturbation Theory for Chain Molecules. Ind. Eng. Chem. Res. 2001, 40, 1244-1260.

Hirschfelder, J.O.; Curtiss, C.F.; Bird, R.B. Molecular Theory of Gases and Liquids; John Wiley \& Sons, Inc.: New York, 1964; pp 1212-1213; oxygen and $n$-heptane from pp 1111-1112.

Tan, S. P.; Kargel, J. S.; Marion, G. M. Titan's Atmosphere and Surface Liquid: New Calculation Using Statistical Associating Fluid Theory. Icarus 2013, 222, 53-72.

Tan, S.P.; Piri, M. Equation-of-state modeling of confined-fluid phase equilibria in nanopores. Fluid Phase Equilib. 2015, 393, 48-63. 


\section{Experimental data of ethane in MCM-41}

The following tables list the experimental data of ethane in MCM-41 porous media, as shown in Fig. 9a of the main paper.

Table S2. Isotherms of ethane in MCM-41 (pore sizes L: $8.08 \mathrm{~nm}$; M: $4.19 \mathrm{~nm}$; S: $2.90 \mathrm{~nm}$ )

\begin{tabular}{|c|c|c|c|c|c|}
\hline \multicolumn{6}{|l|}{272.35 K } \\
\hline \multicolumn{2}{|l|}{ Pore size L } & \multicolumn{2}{|l|}{ Pore size M } & \multicolumn{2}{|l|}{ Pore size S } \\
\hline $\mathrm{P}$ [bar] & Amount [g] & $\mathrm{P}[\mathrm{bar}]$ & Amount [g] & $\mathrm{P}[\mathrm{bar}]$ & Amount [g] \\
\hline 6 & 0 & 2.5 & 0 & 0 & 0 \\
\hline 6.323336 & 1.739587 & 2.800413 & 1.195042 & 0.356344 & 1.327783 \\
\hline 6.59051 & 3.602985 & 3.091541 & 2.707802 & 0.681879 & 2.873714 \\
\hline 7.110833 & 7.269682 & 3.359929 & 4.30915 & 0.923177 & 4.223987 \\
\hline 7.40024 & 9.531937 & 3.635162 & 6.011598 & 1.114995 & 5.315886 \\
\hline 7.727678 & 11.84302 & 3.895979 & 7.639038 & 1.353885 & 6.693974 \\
\hline 8.06544 & 14.02066 & 4.171911 & 9.297888 & 1.514615 & 7.707549 \\
\hline 8.195843 & 14.6839 & 4.309338 & 10.0845 & 1.692915 & 8.686445 \\
\hline 8.280016 & 15.33897 & 4.448922 & 10.84846 & 1.812192 & 9.327979 \\
\hline 8.392033 & 15.99972 & 4.584177 & 11.52661 & 1.931606 & 9.916711 \\
\hline 8.516986 & 16.59617 & 4.709263 & 12.11744 & 2.059033 & 10.49828 \\
\hline 8.621247 & 17.16782 & 4.837166 & 12.6492 & 2.187202 & 11.0235 \\
\hline 8.666417 & 17.65788 & 4.96357 & 13.12836 & 2.308942 & 11.52619 \\
\hline 8.771697 & 18.16196 & 5.096196 & 13.60453 & 2.435007 & 12.02466 \\
\hline 8.863553 & 18.57601 & 5.22499 & 14.12553 & 2.55551 & 12.52574 \\
\hline 8.993695 & 18.88673 & 5.353812 & 14.74501 & 2.675961 & 13.06454 \\
\hline 9.127209 & 19.13483 & 5.470696 & 15.45669 & 2.788914 & 13.66501 \\
\hline 9.231487 & 19.36427 & 5.584145 & 16.22619 & 2.897332 & 14.36054 \\
\hline 9.345745 & 19.63021 & 5.68927 & 17.02334 & 3.04725 & 15.43202 \\
\hline 9.450444 & 19.89603 & 5.883249 & 18.51778 & 3.182517 & 16.48218 \\
\hline 9.570884 & 20.21405 & 6.069558 & 19.91456 & 3.345949 & 17.85838 \\
\hline 9.661362 & 20.69992 & 6.232988 & 21.26838 & 3.463339 & 18.72711 \\
\hline 9.745624 & 21.18227 & 6.412243 & 22.35377 & 3.615761 & 19.9436 \\
\hline 9.824509 & 21.60107 & 6.579981 & 23.31491 & 3.798784 & 21.23008 \\
\hline 9.921851 & 22.09213 & 6.930777 & 23.90351 & 3.952707 & 22.2828 \\
\hline 10.01646 & 22.66251 & 7.467404 & 24.04171 & 4.141951 & 23.35431 \\
\hline 10.16851 & 23.28075 & & & 4.267163 & 23.67051 \\
\hline 10.27485 & 23.54786 & & & 4.569373 & 23.82846 \\
\hline 10.72079 & 23.94542 & & & 5.083697 & 23.93098 \\
\hline 11.37334 & 24.00605 & & & & \\
\hline
\end{tabular}


Table S2 - continued

\begin{tabular}{|c|c|c|c|c|c|}
\hline \multicolumn{6}{|l|}{$267.45 \mathrm{~K}$} \\
\hline \multicolumn{2}{|l|}{ Pore size L } & \multicolumn{2}{|l|}{ Pore size M } & \multicolumn{2}{|l|}{ Pore size S } \\
\hline $\mathrm{P}$ [bar] & Amount [g] & $\mathrm{P}$ [bar] & Amount [g] & $\mathrm{P}$ [bar] & Amount [g] \\
\hline 6 & 0 & 2.5 & 0 & 0 & 0 \\
\hline 6.303699 & 1.626236 & 2.85847 & 1.221508 & 0.289764 & 0.906246 \\
\hline 6.559579 & 3.3824 & 3.126555 & 2.547196 & 0.583526 & 2.115245 \\
\hline 6.776201 & 5.031519 & 3.41677 & 4.121485 & 0.788654 & 3.117355 \\
\hline 7.002115 & 6.764635 & 3.662237 & 5.545275 & 1.043498 & 4.413078 \\
\hline 7.330379 & 9.1232 & 3.883658 & 6.847197 & 1.287817 & 5.706536 \\
\hline 7.687335 & 11.42941 & 4.130252 & 8.211553 & 1.490409 & 6.77464 \\
\hline 7.832032 & 12.10875 & 4.27031 & 8.954528 & 1.658375 & 7.67201 \\
\hline 8.167227 & 14.01955 & 4.514088 & 10.10843 & 1.906646 & 8.788617 \\
\hline 8.35996 & 15.14298 & 4.760657 & 11.03422 & 2.051949 & 9.348823 \\
\hline 8.593365 & 16.0656 & 4.900989 & 11.46781 & 2.190946 & 9.889998 \\
\hline 8.833639 & 16.65724 & 5.169209 & 12.347 & 2.317366 & 10.3404 \\
\hline 8.957988 & 16.86125 & 5.304081 & 12.98526 & 2.44945 & 10.80681 \\
\hline 9.064701 & 17.15205 & 5.417463 & 13.70257 & 2.560119 & 11.29409 \\
\hline 9.19524 & 17.37707 & 5.529697 & 14.50461 & 2.731855 & 12.12443 \\
\hline 9.320274 & 17.60094 & 5.741359 & 16.08145 & 2.878979 & 13.13928 \\
\hline 9.438137 & 18.1134 & 5.91609 & 17.52197 & 3.042439 & 14.42474 \\
\hline 9.528906 & 18.62108 & 6.100156 & 18.88243 & 3.215946 & 15.83722 \\
\hline 9.700841 & 19.58759 & 6.289495 & 20.09845 & 3.399852 & 17.34623 \\
\hline 9.847084 & 20.38554 & 6.488121 & 20.77619 & 3.653206 & 19.27334 \\
\hline 9.980113 & 20.72494 & 6.693804 & 21.04551 & 3.809233 & 20.31414 \\
\hline 10.27246 & 21.00909 & & & 3.911988 & 20.75187 \\
\hline 10.91756 & 21.21387 & & & 4.143242 & 20.92768 \\
\hline \multicolumn{6}{|l|}{$262.45 \mathrm{~K}$} \\
\hline \multicolumn{2}{|l|}{ Pore size L } & \multicolumn{2}{|c|}{ Pore size M } & \multicolumn{2}{|l|}{ Pore size S } \\
\hline $\mathrm{P}$ [bar] & Amount [g] & $\mathrm{P}[\mathrm{bar}]$ & Amount [g] & $\mathrm{P}[\mathrm{bar}]$ & Amount [g] \\
\hline 6 & 0 & 2.5 & 0 & 0 & 0 \\
\hline 6.277017 & 1.262517 & 2.851384 & 1.177607 & 0.320433 & 0.852292 \\
\hline 6.566608 & 2.968422 & 3.12399 & 2.407273 & 0.635498 & 2.033182 \\
\hline 6.833658 & 4.71974 & 3.392284 & 3.779803 & 0.964063 & 3.468783 \\
\hline 7.104521 & 6.457201 & 3.668415 & 5.2164 & 1.155882 & 4.388203 \\
\hline 7.335301 & 7.842719 & 4.050741 & 7.269072 & 1.377369 & 5.484411 \\
\hline 7.503607 & 9.099005 & 4.30619 & 8.445391 & 1.61999 & 6.583067 \\
\hline 7.622716 & 9.851002 & 4.56181 & 9.446815 & 1.831617 & 7.4504 \\
\hline 7.812831 & 11.03817 & 4.833068 & 10.15123 & 2.068165 & 8.286537 \\
\hline 8.011921 & 12.15003 & 4.975156 & 10.50124 & 2.245518 & 8.821219 \\
\hline 8.258064 & 13.205 & 5.124266 & 10.96376 & 2.497733 & 9.585544 \\
\hline 8.381254 & 13.71605 & 5.253586 & 11.60236 & 2.676704 & 10.3289 \\
\hline 8.629328 & 14.38439 & 5.378531 & 12.38013 & 2.810989 & 11.21164 \\
\hline 8.767363 & 14.59415 & 5.49823 & 13.19148 & 2.934582 & 12.1931 \\
\hline 8.892873 & 14.79295 & 5.706988 & 14.88917 & 3.082416 & 13.4165 \\
\hline 9.020139 & 14.99794 & 5.916934 & 16.40567 & 3.217447 & 14.60763 \\
\hline 9.161416 & 15.16869 & 6.112261 & 17.6984 & 3.355935 & 15.86477 \\
\hline 9.271251 & 15.35757 & 6.348688 & 18.41603 & 3.498178 & 17.06135 \\
\hline 9.385459 & 15.83244 & 6.617391 & 18.65879 & 3.603747 & 17.8268 \\
\hline 9.476717 & 16.34921 & 7.006704 & 18.71377 & 3.690695 & 18.2286 \\
\hline 9.654046 & 17.3611 & 7.55048 & 18.85731 & 3.852643 & 18.40436 \\
\hline 9.80971 & 18.0574 & 7.809788 & 18.90246 & 4.045813 & 18.51907 \\
\hline 9.879471 & 18.29314 & 8.481575 & 18.99067 & 4.691945 & 18.64011 \\
\hline 10.0507 & 18.48425 & & & 4.995309 & 18.6899 \\
\hline 10.24125 & 18.58476 & & & 5.569624 & 18.77464 \\
\hline 10.55682 & 18.70744 & & & & \\
\hline
\end{tabular}


Table S2 - continued

\begin{tabular}{|c|c|c|c|c|c|}
\hline \multicolumn{6}{|l|}{$257.15 \mathrm{~K}$} \\
\hline \multicolumn{2}{|l|}{ Pore size L } & \multicolumn{2}{|c|}{ Pore size M } & \multicolumn{2}{|l|}{ Pore size S } \\
\hline$P[$ bar $]$ & Amount [g] & $\mathrm{P}[\mathrm{bar}]$ & Amount $[g]$ & $\mathrm{P}[$ bar $]$ & Amount [g] \\
\hline 6 & 0 & 2.5 & 0 & 0 & 0 \\
\hline 6.116843 & 0.475693 & 2.714984 & 0.471588 & 0.218219 & 0.641253 \\
\hline 6.340124 & 1.544719 & 2.860848 & 0.968091 & 0.387262 & 1.207996 \\
\hline 6.530264 & 2.5526 & 3.05836 & 1.742094 & 0.584192 & 1.98223 \\
\hline 6.896663 & 4.834633 & 3.329113 & 2.959334 & 0.779551 & 2.859611 \\
\hline 7.135625 & 6.330765 & 3.564939 & 4.087658 & 0.979827 & 3.685988 \\
\hline 7.379936 & 7.759198 & 3.861844 & 5.518908 & 1.128816 & 4.283924 \\
\hline 7.514897 & 8.509662 & 4.140627 & 6.804749 & 1.264309 & 4.848084 \\
\hline 7.811259 & 9.85851 & 4.288323 & 7.406858 & 1.400095 & 5.382915 \\
\hline 7.874854 & 10.49577 & 4.562945 & 8.275793 & 1.605961 & 6.107562 \\
\hline 8.0164 & 11.11209 & 4.704153 & 8.535139 & 1.786886 & 6.671999 \\
\hline 8.148938 & 11.66114 & 4.863275 & 8.832316 & 1.926684 & 7.031444 \\
\hline 8.294834 & 12.09672 & 5.018424 & 9.17646 & 2.069864 & 7.38371 \\
\hline 8.423587 & 12.36453 & 5.166733 & 9.769403 & 2.22814 & 7.782606 \\
\hline 8.556922 & 12.54639 & 5.298008 & 10.56863 & 2.398877 & 8.329696 \\
\hline 8.694324 & 12.72959 & 5.42656 & 11.48607 & 2.555234 & 9.185993 \\
\hline 8.845082 & 12.9307 & 5.553188 & 12.40557 & 2.671831 & 10.03967 \\
\hline 8.976574 & 13.10438 & 5.666928 & 13.32409 & 2.783882 & 11.02042 \\
\hline 9.111671 & 13.31421 & 5.783255 & 14.19841 & 2.919607 & 12.10688 \\
\hline 9.231474 & 13.84439 & 5.900698 & 14.97353 & 3.037164 & 13.15657 \\
\hline 9.425954 & 14.84734 & 6.139898 & 15.8621 & 3.149501 & 14.02465 \\
\hline 9.608948 & 15.68596 & 6.387008 & 16.13452 & 3.247321 & 14.77491 \\
\hline 9.747132 & 15.97935 & 6.765388 & 16.26728 & 3.331623 & 15.35723 \\
\hline 10.15996 & 16.17426 & 7.249156 & 16.37778 & 3.600283 & 15.78568 \\
\hline 10.65803 & 16.31354 & 7.705681 & 16.44197 & 4.056119 & 15.89306 \\
\hline 11.21808 & 16.39486 & & & 4.583207 & 16.01856 \\
\hline \multicolumn{6}{|l|}{$252.15 \mathrm{~K}$} \\
\hline \multicolumn{2}{|l|}{ Pore size L } & \multicolumn{2}{|l|}{ Pore size M } & \multicolumn{2}{|l|}{ Pore size S } \\
\hline$P$ [bar $]$ & Amount [g] & $\mathrm{P}$ [bar] & Amount [g] & $\mathrm{P}[\mathrm{bar}]$ & Amount [g] \\
\hline 6 & 0 & 2.5 & 0 & 0 & 0 \\
\hline 6.318472 & 1.031876 & 2.89092 & 1.034372 & 0.267004 & 0.888966 \\
\hline 6.452897 & 1.725862 & 3.003159 & 1.43279 & 0.481024 & 1.576317 \\
\hline 6.559463 & 2.289601 & 3.158487 & 1.886354 & 0.630066 & 2.122111 \\
\hline 6.631747 & 2.790476 & 3.345459 & 2.92617 & 0.856681 & 2.965527 \\
\hline 6.727923 & 3.368885 & 3.531439 & 3.845935 & 1.037112 & 3.786869 \\
\hline 6.828851 & 3.936113 & 3.62111 & 4.301541 & 1.262048 & 4.589442 \\
\hline 7.008177 & 4.518758 & 3.735309 & 4.73608 & 1.462086 & 5.291116 \\
\hline 7.135407 & 5.28778 & 3.929114 & 5.552871 & 1.612338 & 5.715267 \\
\hline 7.265467 & 6.045735 & 3.985619 & 5.820607 & 1.761496 & 6.079107 \\
\hline 7.396833 & 6.792885 & 4.18325 & 6.534168 & 1.979971 & 6.598023 \\
\hline 7.660112 & 8.213456 & 4.264736 & 6.840525 & 2.121801 & 6.905201 \\
\hline 7.81297 & 9.032047 & 4.402395 & 7.096877 & 2.330618 & 7.369495 \\
\hline 8.12166 & 10.37638 & 4.595561 & 7.458208 & 2.506501 & 8.041129 \\
\hline 8.382136 & 11.05571 & 4.781295 & 7.715942 & 2.573533 & 8.40512 \\
\hline 8.648174 & 11.34998 & 4.93056 & 8.108775 & 2.685483 & 9.21887 \\
\hline 8.916485 & 11.72047 & 5.111047 & 8.85162 & 2.778715 & 9.972497 \\
\hline 9.095137 & 11.98795 & 5.195057 & 9.374855 & 2.924511 & 11.1679 \\
\hline 9.177148 & 12.32475 & 5.326097 & 10.34203 & 3.094868 & 12.63064 \\
\hline 9.385833 & 13.41509 & 5.450049 & 11.31621 & 3.237376 & 13.62674 \\
\hline 9.476977 & 13.83151 & 5.597184 & 12.4118 & 3.285845 & 13.91717 \\
\hline 9.51091 & 13.97405 & 5.734241 & 13.35177 & 3.411128 & 14.30584 \\
\hline 9.574211 & 14.17569 & 5.99716 & 14.22312 & & \\
\hline 9.710335 & 14.42092 & 6.281671 & 14.41446 & & \\
\hline 10.67671 & 14.69811 & 6.733254 & 14.51451 & & \\
\hline & & 7.24384 & 14.57785 & & \\
\hline
\end{tabular}


Table S2 - continued

\begin{tabular}{|c|c|c|c|c|c|}
\hline \multicolumn{6}{|l|}{$247.25 \mathrm{~K}$} \\
\hline \multicolumn{2}{|l|}{ Pore size L } & \multicolumn{2}{|l|}{ Pore size M } & \multicolumn{2}{|l|}{ Pore size S } \\
\hline $\mathrm{P}$ [bar] & Amount [g] & $\mathrm{P}$ [bar] & Amount [g] & $\mathrm{P}$ [bar] & Amount [g] \\
\hline 6 & 0 & 2.5 & 0 & 0 & 0 \\
\hline 6.179481 & 0.491986 & 2.768172 & 0.4894 & 0.274234 & 0.47169 \\
\hline 6.356709 & 1.195986 & 2.917681 & 0.925053 & 0.46417 & 0.940924 \\
\hline 6.510877 & 1.871529 & 3.131511 & 1.607986 & 0.617974 & 1.370949 \\
\hline 6.609957 & 2.359654 & 3.254536 & 2.143856 & 0.807476 & 2.006178 \\
\hline 6.806058 & 3.383986 & 3.445135 & 2.863704 & 0.957519 & 2.501875 \\
\hline 7.008938 & 4.359886 & 3.681904 & 3.709768 & 1.152849 & 3.168043 \\
\hline 7.22896 & 5.557082 & 3.829638 & 4.421345 & 1.335198 & 3.767558 \\
\hline 7.402565 & 6.409197 & 3.961962 & 4.874614 & 1.521299 & 4.33596 \\
\hline 7.670702 & 7.56306 & 4.254197 & 5.779375 & 1.733702 & 4.907294 \\
\hline 7.835433 & 8.263141 & 4.42031 & 6.085137 & 1.932195 & 5.306239 \\
\hline 7.995837 & 8.833036 & 4.595565 & 6.301077 & 2.07622 & 5.552071 \\
\hline 8.238515 & 9.298804 & 4.795644 & 6.527391 & 2.201675 & 5.763015 \\
\hline 8.393296 & 9.460475 & 4.979259 & 6.980557 & 2.343176 & 5.990144 \\
\hline 8.655407 & 9.745655 & 5.149728 & 7.823894 & 2.465835 & 6.277906 \\
\hline 8.856268 & 9.921854 & 5.288543 & 8.835838 & 2.597368 & 6.756592 \\
\hline 9.014162 & 10.41143 & 5.505792 & 10.45807 & 2.705889 & 7.387435 \\
\hline 9.155952 & 10.97785 & 5.70411 & 11.61299 & 2.84428 & 8.528566 \\
\hline 9.228762 & 11.46006 & 5.776713 & 11.8634 & 3.012812 & 9.865565 \\
\hline 9.351866 & 11.88638 & 6.105646 & 12.27868 & 3.170871 & 11.38582 \\
\hline 9.437608 & 12.16057 & 6.595818 & 12.38792 & 3.261379 & 11.86564 \\
\hline 9.621964 & 12.42497 & 6.984165 & 12.48909 & 3.357947 & 12.09847 \\
\hline 10.89766 & 12.78048 & & & 3.778522 & 12.39469 \\
\hline \multicolumn{6}{|l|}{$242.25 \mathrm{~K}$} \\
\hline \multicolumn{2}{|l|}{ Pore size L } & \multicolumn{2}{|l|}{ Pore size M } & \multicolumn{2}{|l|}{ Pore size S } \\
\hline $\mathrm{P}$ [bar] & Amount $[g]$ & $\mathrm{P}$ [bar] & Amount [g] & $\mathrm{P}$ [bar] & Amount [g] \\
\hline 6 & 0 & 2.5 & 0 & 0 & 0 \\
\hline 6.212798 & 0.533978 & 2.805041 & 0.530404 & 0.240347 & 0.348672 \\
\hline 6.384087 & 1.157516 & 3.299611 & 1.961054 & 0.462047 & 0.816114 \\
\hline 6.60754 & 2.243182 & 3.45393 & 2.536386 & 0.688577 & 1.381031 \\
\hline 7.037916 & 4.115168 & 3.587005 & 3.011616 & 0.923099 & 2.064209 \\
\hline 7.429901 & 6.005071 & 3.955227 & 4.165977 & 1.082866 & 2.526673 \\
\hline 7.735006 & 6.979998 & 4.134831 & 4.765904 & 1.277936 & 3.106356 \\
\hline 7.954814 & 7.494164 & 4.321516 & 5.108516 & 1.472468 & 3.653976 \\
\hline 8.048842 & 7.807111 & 4.563207 & 5.347816 & 1.691475 & 4.146082 \\
\hline 8.254626 & 7.982251 & 4.808316 & 5.601952 & 1.897831 & 4.508764 \\
\hline 8.404635 & 8.137476 & 5.011426 & 6.120043 & 2.129929 & 4.797914 \\
\hline 8.665773 & 8.380419 & 5.22958 & 7.411943 & 2.322138 & 5.109615 \\
\hline 8.882217 & 8.636398 & 5.463034 & 9.103889 & 2.4549 & 5.418406 \\
\hline 9.160927 & 9.68789 & 5.638383 & 10.10897 & 2.694993 & 6.608016 \\
\hline 9.355989 & 10.22316 & 5.882159 & 10.55219 & 2.798351 & 7.518544 \\
\hline 9.389644 & 10.4015 & 6.104109 & 10.69243 & 2.962968 & 8.640546 \\
\hline 9.650323 & 10.68659 & 6.590209 & 10.78579 & 3.160118 & 10.09936 \\
\hline 10.11936 & 10.83306 & 7.133531 & 10.88708 & 3.206166 & 10.3331 \\
\hline & & & & 3.316204 & 10.49434 \\
\hline
\end{tabular}

